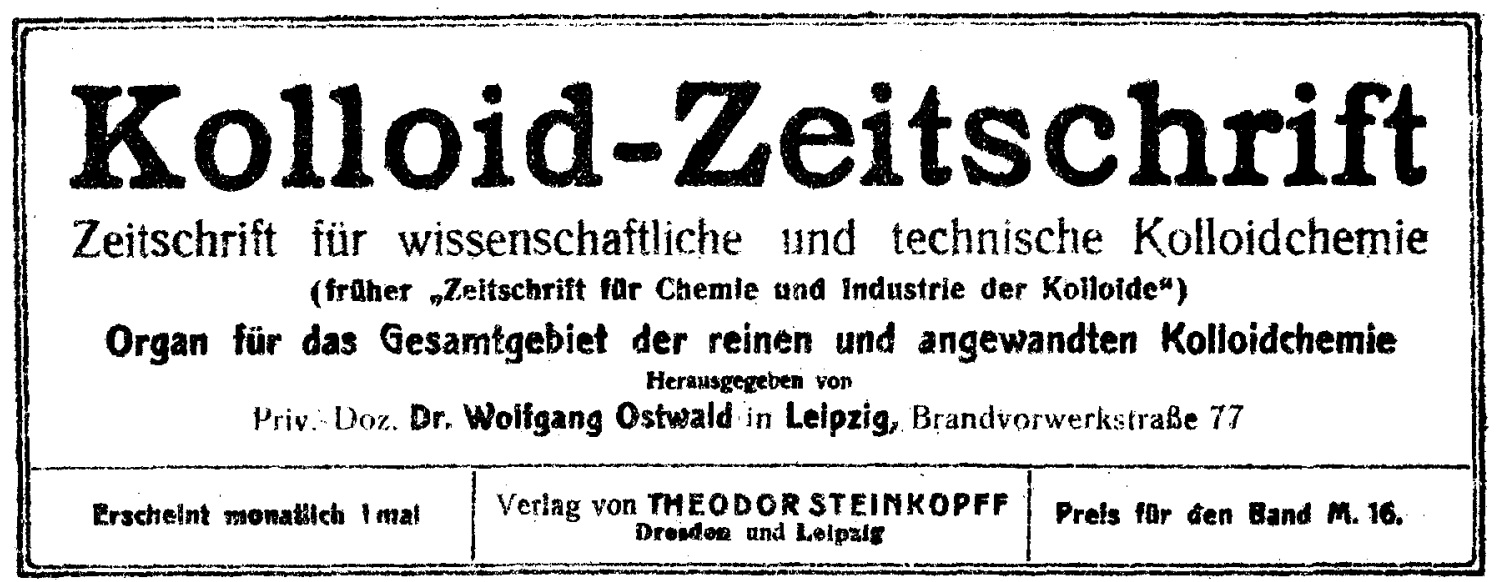

\title{
Niederschlags- und Schichtenbildung in Abwesenheit von Gelen.
}

Von W. P. Dreaper (London).

( Hingegangan 2m if. Fobmar 1914)

Obgleich die Gegenwart von Gelen für die Schichtbildung wesentlich sein bsann, wenn die Strömung von Lösungen nicht kontrolliert wird, so labt sich doch zeigen, dati unter den in folgenden: zu beschreibenden Bedingungen in gleicher Weise charakteristische Reaktionen in ihrer Abwesenheit beobachtet werden konnen.

Die in folgenden $z u$ beschreihenden Versuche wurden ausgefuhrt, 1 bei Fallung innerhalb vor Kapillarröhren, die nicht mehr als 0,1 Millimeter Durchmesser besitzen, und 2. bei Pälung in Gegenwart gewissè permeabler Schichten, wie Sand oder Kieselgur.

Was die Kapillarröhren anbetrifft, so hat es sich als möglich herausgestellt, die infolge ungleichmäBiger Diffusion aufretenden störenden Einflisse, Gegenströmungen, die Beeinflussung durch die Schwerkratt und abnliches auszuschalten.

Für die Zwecke der Untersuchung hat es sich als notwendig erwiesen, einen Unterschied zu machen zwischen den Resultaten, die in Gegenwart von zugesetzten Gien erhalten wurden, und denjenigen, wo Gelbilduig als Produkt der Reaktion eingetreten war. Demzufolge werden die latzieren auch als „primäre" Gele und die zu den lösungen zugesetzter als ; sckundäre" Gele bezeichnet.

Die für die Untersuchung bexutzue Apparatur ist ziemlich einfach. Ein Kapillarrohr von ungefähr $20 \mathrm{cni}$ Lisnge wird an einem Ende rechtwinklig derart gebogen, dab der kürzete Schenk.! ungefihti. $3 \mathrm{~cm}$ lang ist.

1) Deutsch won H. Brehm (Dresden)
Darauf wird das Rohr mit einer der Re. aktionslösungen mittels Kapillarwirkung be. schickt und das umgebogene Ende in der Weise abgeschmolzen, daB das Rohr nach erfolgter Abkühlung völlig mit der Lösung angefüllt ist.

Fin anderes Rohr von 2 bis 3 Millimeter Durchmesser wird durch Blasen an einem Ende mit einer Kugel oon ungefähr $2 \mathrm{~cm}$ Durchmesser versehen; die gesamte länge beträgt etwa $16 \mathrm{~cm}$. Es wird $z u 10 \mathrm{~cm}$ seinet Länge mit der zweiten Reaktionsiösung gefinll. Darauf wird dieses zweite Rohr in horizontaler Lage festgeklemmt, dann das Kapillarrohr in das offene Ende deaselben vorsichtig so weit eingerührt, bis es mit seinem offenen Ende in die Mitte der Kugel des äutberen Rohres gelangt ist. In dieser Lage zueinander werden die beiden Rohre bis zur Beendigung des Versuches festgehalten. Um Verdunstung aus den lösungen zâ verhindern, läßi man auf das offene Ende des áufieren Rohres etwas Wachs tropfen. Auf diese Weise kann man diese Versuche bis zu einer Dauer von mehreren Monaten ausdehnen, ohne dab Aenderumgen der Konzentration der loosungssysteme innerhalb der Rohren eintreten können.

Einige von den erhaltenen Resultaten sind veröffenthich worden im Journ. Soc. Chem. Ind. (32, $677[1913])$ sowie in aer Chemical World $(2,343[1913])$.

Hier möge zunächst ein Versuch beschrieben werden, bei dem das Kapillarrohr eine dreiprozcntige Lösung von Bleiazetat und das atufere Rohr eine fünfprozentige Lösung von Cricrwasserstoffsäure $(\mathrm{HCl})$ enthielt. Es bilden 
sich in diesem Falle in dem Kapillarrohr langsam Kristalle von Bleichlorid; eine Anzahl von einzelnen Kristallen setzte sich in zunehmenden $Z$ wischenrätimen voneinander aufwärts des Kapillarrohrs ab. Die wirklichen Entfernungen der Kristalle voneinander sind aus der folgender Tabelle zu ersehen, und ihre relative Lage kann vergilichen werden mit den Schichtbildungsresultaten, die in Rohren von gröBerem Durchmesser in Gegenwart von Gelen erhaiten worden sind. Wenn man die ersten beiden Resultate nicht mit berücksichtigt wegen der an offenen Ende des Kapillarrohrs auftretenden störenden Einflüsse, so geben die erhaltenen Zahlen eine geradlinige Kurve, sobald man den Logarithmus des Abstandes zwischen den Mittelpunkten zweier aufeinanderfolgender Kristalle und anderseits den Logarithmus des Abstands ihrer Mittelpunkte vom Ende des Kapillarrohrs abträgt. Dieser Effekt ließ sich jedoch nicht immer erzielen.

Tabelle A

Bleichlorid im Kapillarrohr. Lumen $0,66 \mathrm{~mm}$.

\begin{tabular}{|c|c|c|}
\hline $\begin{array}{c}\text { Länge } \\
\text { der } \\
\text { Kristalle } \\
\text { mm } \\
\end{array}$ & $\begin{array}{l}\text { Abstand yon } \\
\text { Mittelpunkt zu } \\
\text { Mittelpunkt der } \\
\text { Ktistalle } \\
\text { mm }\end{array}$ & $\begin{array}{c}\text { Wirklicher } \\
\text { Zwischenraum } \\
\text { zwischen den } \\
\text { Kristallen } \\
\text { unm } \\
\end{array}$ \\
\hline 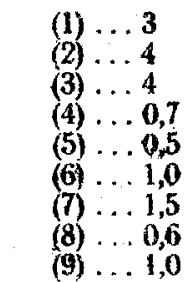 & $\begin{array}{lll}(1-2) & \ldots & 6,7 \\
(2-3) & \ldots & 8 \\
(3-4) & \ldots & 5,75 \\
(4-5) & \ldots & 5,85 \\
(5-6) & \ldots & 6,0 \\
(6-7) & \ldots & 7,75 \\
(7-8) & \ldots & 7,05 \\
(8-9) & \ldots & 8,2 \\
(9-10) & \ldots & 11,75\end{array}$ & $\begin{array}{ll}(1--2) & \ldots 3 \\
(2-3) & \ldots 4 \\
(3-4) & \ldots 2,5 \\
(4-5) & \ldots 3,3 \\
(5-6) & \ldots 5,5 \\
(6-7) & \ldots 6,5 \\
(7-8) & \ldots 6,5 \\
(8-9) & \ldots 7,5 \\
(9-10) & \ldots 9,0\end{array}$ \\
\hline
\end{tabular}

Die von den Bleikristallen angenommene Kristallform ist anormal. In Figur 1 und 2 (Taf. I) sind die den Nummern 6 und 7 der vorstehenden Tabelle entsprechenden Kristalle, die in situ photographiert wurden, abgebildet.

Der rechtwinklig gebogene Schenkel hat den Zweck, daB das Kapillarrohr aus dem äuberen Rohr herausgenommen; photographiert und dann wieder in gleiche Lage an Ort und Stelle gebracht werden kann. Die an den rechtwinklig abgebogenen Schenkel angreifende Schwerkraft bringt die Rohre wieder genau in die gleiche Lage zurück.

An dieser Stelle möge bei der Geiegenheit erwähnt werden, daß die yon den Kristallen in bezug zur Richtung des Eintritts der zweiten Lösung (durch Pfeil gekennzeichnet) angenom- mene besondere form vielleicht dazu benutzt werden kann, um ăhnliche Verhältnisse klar zu steilen, die sich möglicherweise in der Natur in der Vergangenheit abgespielt haben.

Wenn man in obigem Versuch die Chlorwasserstoffsäure durch Kaliumferrozyanid ersetzt, so lassen sich gut ausgebildete Kristalle von Bleiferrozyanid leicht erhaiten. Ihre Form weicht jedoch ab von der durch E. Hatschek in Gegenwart von Gelen beobachteten, und vielleicht lassen sich derartige Unterschiede ebenfalls zur Kennzeichnung von Bedingungen benutzen, wie sie in der Vergangenheit bei der Bildung gewisser mineralischer Ablagerungen etwa ebenfalis vorgelegen haben. Bis jetzt sind Sphärolithe jedoch in diesen Rohren noch nicht beobachtet worden.

Bei Gegenwart von Schwefelsäure ader Káliumsulfat im äuBeren Rohr und von Bleiazetat in inneren läht sich eine deutliche Schichtbildung erzielen. Das gebildete Bleisulfat tritt dabei im allgemeinen in einem äuberst. feinverteilten Zustande auf. Dasselbe ist so kleinkörnig, daB der Niederschlag über den Querschnitt des Kapillarrohres lange Zeit gleichmäbig verteilt bleibt. Sein physikalischer Zustand scheint sich beinahe dem eines Kolloids im Gelzustande zu nähern. Erst nach Verlauf mehrerer Monate macht sich eine Neigung des Niederschlags zum Absetzen auf den Boden des Rohrs bemerkbar. Der bei Eintritt dieses Zustandes hervorgebrachte Effekt ist in Fig. 3 wiedergegeben, wo, wie man sieht, die ursprüngliche Schichtbildung zum Teil zerstört ist. Der Niederschlag ist jetzt völlig kristallinisch geworden. In einem anderen Falle, in dem die Fällung sich sehr langsam vollzog, fiel das Bleisulfat sofort. in völlig kristallinischem Zustande aus, wie in Fig. 4 abgebildet ist. Die großen $0,8^{\circ} \mathrm{mm}$ langen Kristalle bildeten sich in beträchtlicher Entfernung aufwärts in dem Kapillarrobr, während die dunklen Massen des feinverteilten Niederschlags am offenen Ende des Rohres entstanden.

In einigen Făllen beobachtete man, dak der zuerst gebildete Niederschlag in derselben Weise wie ein zugesetztes Gel wirkte, indem er innerhalb seines Entstehungsbereiches eine darauffolgende Schichtung herbeiführte. So z. B. wurde, wenn das Kapillarrohr eine zweiprozentige Lösung von Bariumchlorid und das äußere Rohr eine fünfprozentige Lösung von Kaliumsulfat enthielt, ein halbopaker Niederschlag aufwärts im Rohr in ungefähr $80 \mathrm{~mm}$ Entfernung zuerst abgesetzt. Bei weiterer Bin- 
wirkting lie sich beobachten; dag eine Neigung zum weiteren Absatz von Bariumsulfat in geschichteten Lagen innerhalb dieses Fällungsgebietes bestand. Diese Absätze können ganz genau begrenzt sein. Die zuerst gebildete $\mathrm{Ab}$ lagerung, die ihrem Aussehen nach einem Gel ähnelt, bleibt gleichinäfig verteilt über den ganzen Querschnitt des Kapillarrohrs und ist nahezu durchsichtig. Es ist $z$. B. beobachtet worden, dab unter gewissen Bedingungen der zuerst gebildete Niederschlag innerhalb seines eigenen Entstehungsgebietes eine darauffolgende Schichtbildung hervorrufen kann. Auch dieses Verhalten kann ganż offensichtlich eine gewisse Rolle spielen bei der Bildung yon natürlichen Mineralablagerungen.

Die mit Bariumkarbonat erhaltenen Ërgebnisse zeigen Schichtbildung und der Niederschlag zeigt hier keine Tendenz, sich auf dem Boden des Rohrs abzusetzen. Das kann man aus Fig. 5 sehr deutlich ersehen; diese Mikrophotographie ist drei Monate nach Bildung des Niederschlages aufgenommen. Auch in dern Zwischenrâumen hatte sich in diesem Falle absolut kein Niederschlag von Bariumkarbonat gebildet.

Aus den Resultaten dieser und anderer Versuche glaubte man die Möglichkeit herleiten zu dürfen, daß der Durchmesser des Kapillarrohres vergrößert werden könne, wenn ein poröses aber reaktionsunfähiges Material, wie z. B. Sand oder Kieselgur zugegen wäre, und $z$ war sobald die Zwischenrătime $z$ wischen den Sandteilchen über den Durchmesser des Kapillarrohrs nicht hinausgingen. Diese Vermutung besätigte sich auch und man. kam zu der Ansicht, daB dieses Resultat eine gewisse- Bedeutung fïr die Art der bei der Bildung von Mineralablagerungen etwa vorkommenden $\mathrm{Be}$ dingungen haben könnte.

In diesem Palle gestaltete die Arbeitsmethode sich etwas abweichend. Ein $12 \mathrm{~cm}$ langes Glasrohr mit einem Durchmesser von $3 \mathrm{~mm}$ wird auf eine Strecke von $7 \mathrm{~cm}$ vom einen Ende mit feinem Sand oder Kieselgur beschickt, die dann durch einen schon vorher in das untere Ende eingeschobenen Pfropfen von Filtrierpapier an ihrem Platze gehalten werden. Die so beschickte Kapillarröhre wird in ein gewöhnliches Reagenzrohr eingebracht, welch letzteres die zur Săttigung der Kieselgurschicht erforderliche Lösung enthalt. Diese Sättigung vollzieht sich durch Kapillaritätswirkung, hierauf wird das Kapillartohtr ats dem Reagenzglas genommen und dan zur Aus- fuhrung des Versuchs geschritten. Zu diesen Zweck wird das Rohr in dasjenige, welches die erste Lossung enthält, gebracht und darn die zweite Lösung vorsichtig und langsam in das obere Ende des inneren Rohres eingegossen, so dab sie über der Säule von Kiegelgur steht. Dann wird der Spiegel der äußeren Lösung mit der Lösung vom inmeren Rohr in gleiche Höhe gebracht, so da auf diese Weise die Störung der Resultate durch ungleichnatige Diffusion verhindert wird.

Beim Arbeiten unter derartigen Bedingungey mit einer zweiprozentigen - Bleiaze tatlösung im Prüfungsrohr und einer zweiprezentigen Lösung von Natriumsuifid, die über der Kieselguhrschicht steht, kann man den in Fig. 6 abgebiideten Effekt erhalten. Inner halb eirier gewissen Strecke abwärts der Kieselg ur schicht bildet sich kein Niederschlag, dann lagert sich ein einzelnes Band von Schwefe l. blei $\mathbf{a b}$. Eine weitere Einwirkung äuBert sich in einer : stärkeren Ablagerung von Schwefelblei innerhalb des Ciebiets dieses Bandes. Eine Schichtbildung konnte jedoch nicht beoba ish tet werden.

Die Ursache dieser Einwirkung ist sehr interessant. Der Raum oberhalb des Bandes von Bleisulfid stellte sich als völlig frei von Bleisalz heraus, welches durch Oberflächenwirkung bei seinem Durchtritt durch die $A$ blagerungszone aus seiner Lossung herausad sorbiert worden war. Erst wenn die herabdiff $u n$ dierende Natriumsulfidlösung einen Punkt erreichte, wo das Btei in dieser Weise noch nicht entfernt worden war, trat eine Fälung von Schwefelblei ein.

Bei Variieren der Fällungsbedingungen wa ren auch einige Fälle darunter, welche eine geschichtete Ablagerung ergaben, wie in Fig: 7 abgebildet ist. Um diesen Effekt zu erzielen, mus man, soweit ich bis jetzt zu übersehen vermag, bestinmte engumgrenzte Bedingungen innehalter, von denen die haupisächlichste de i bildet, dab die Fiässiglkeitsströmung nur nach einer Richtung sich bewegt. Hier ist aber. noch weitere Expierimentalarbeit nötig und daher sollen die wateren finzelheiten bei einer anderen Gelegenheit angegeben werden. Wern man aber die Säsle von Kieselgur 48 Stunden lang in Berühnung mit einem Ueberschub von Blelazetatiosung läBt, so wird das Bleisalz durch die oberste Schicht der Kieselgursäule durchdiffundieren und dann bildet sich sofort ein Niederschlag an itrer Oberfachenschicht. sobold die Natriunsulfilosung zugesetzt wird. 
Nach dieser Einwirkungsperiode wird thas Rohr aus der Bleilösung entfernt und letztere entweder durch Wasser oder Natriumsulfidlossung ersetzt. Darauf wird die Natriumsulfidlösung in das obere Ende des inneren Rohrs gegossen und die beiden Flüssigkeitsoberflachen wie vor. her in gleiche Höhe gebracht. Unter solchen Bedingungen, wo das Bleisalz nicht im Ueberschub vorhanden ist, wird die Schichtbildung erzielt.

Daß die Sulfide der Schwermetalle Schichtbildung in Kapillarrohren ergeben, zeigt sich aus Abb. 8, wo ein Kupfersulfidniederschlag hervorgerufen worden ist; das bohe spezilische Gewicht des Niederschlags ruft den aus der Abbildung zu ersehenden Effekt sehr rasch hervor

Diese Versuche in weiten Rohren, die schon einen verhaitnismäbig groben Durchmesser haben dürfen, lassen dife Vorgänge bei der Schichtbildung in besserer Beleuchtung erkennen und sie stellen möglicherweise diejenigen Bedingungen dar, die in der Natur mit mehr Wahrscheinlichkeit nock als die vorher beschriebenen vorgekommen sein könaten. Unter diesen Verhältnissen werden gerade Sulfide eine Schichtbildung zeigen.

Bei dieser Gelegenheit möchte ich auch noch auf gewisse Resuitate zurückiromen, die ich bei der Anwesenheit won Gelen (Gelatine) mit Rohren von $3 \mathrm{~mm}$ Durchnesser erhalten habe. Denn unter diesen Bedingungen kbnnen die vorkommenden Reaktionen leicht beobachtet werden. So $z$. $\$$. werden im Faile des Bariumsulfats interessante Resultate erhalten. Eine Gelatinelösung, in der a Proz. Batiumchlorid verteilt waren, wurde in eine $3 \mathrm{~mm}$-Röhre eingefüllt. Letztere wurde darauf in eine Lösung von Kaliumsulfat gebracht, so dab sie vollig mit Lösung bedeckt war und die Einwirkung von beiden Enden aus erfolgen konnte. Unter diesen Umständen bestand der erste Effekt in Bildung einer dichten Ablagerung von Bariumsulfat an beiden Enden. Nach einer gewissen Periode war dieselbe bis zu einer Entfernung von $8 \mathrm{~mm}$ von jedern Ende in die Gelatinesätule vorgedrungen. Die weitere Einwirkung gestaltete sich jedoch ganz aubergewöhnlich. Diese Säulen von $8 \mathrm{~mm}$ verlängerten sich nicht mehr, sondern statt dessen schienen sie von beiden Enden aus nach der Mitte des Rohres zu vorzudringen, indem sie einen von Niederschlag freien Raum hinter sich liefien. Dieser Effekt dauerte an, bis sie eine in Fig. 9 abgebildete Stellung erreicht hatten, wobei ein unangegriffenes $Z$ wischenstück von $3 \mathrm{~mm}$ zwischen ihnen übrig biieb. Hierauf rückten sie nicht. mehr weiter yor, sondern verkürzten sich nach und nach und verschwanden schließlich vollständig. Diese Rohre waren so beschaffen, daB man sie leicht an einem beliebigen Punkte abbrechen und ảie Gelatine aus dem betreffenden Abschnit des Rohrs durch gelindes Erwärmen entfernen konnte.

Es stellte sich dann heraus, dab diese Wanderung der Bariumsulfatsäule ein mehr scheimbarer als tatsächlicher Vorgang ist; der tatsăchliche Vorgang bestand darin, daf sich die physikalische und moglicherweise auch die chemische Natur des gefällten Bariumsulfats bei weiterer Einwirkung eines Ueberschusses von Kaliumsulfat völlig veränderte. In Fig. 10 ist das zuerst gebildete Bariumsulfat abgebildet, in Fig. 11 derselbe Niederschlag nach der weiteren Einwirkung von Kaliumsulfat im Ueberschut. Die Ursache der oben beschriebenen scheinbaren Wanderung erklärt sich also hieraus. Das im Zentrum der Gelatinesäule vollig frei bleibende Stuck wurde frei von Barium gefunden; letzteres war vielmehr sämtlich in Richtung auf beide Enden des Rohres fortdiffundiert. Dieser Teil der Vorgänge ist noch etwas dunkel, da weder ein anderer Elektrolyt an die Stelle des Kalisalzes eintreten kann, noch im Ueberschub vorhandenes Bariumchlorid irgendwelche derartige Wirkung haben wird. Deswegen mag sich im Falle von Fig. 11 ein Bariumkalium-Doppelsalz gebildet haben. Das bestätigte sich auch bei näherer Untersuchung. Diese Reaktion ist insofern interessant im Hinblicke auf den Umstand, daB dieses Doppelsalz sich unter gewöhnlichen Umständen nur beim Zusammenschmelzen bildet. 